\title{
Mesures de pressions et de vitesses dans un ventilateur centrifuge (rotor et stator) avant et après l'apparition et la disparition des décollements tournants
}

\author{
Pressure and speed measurements in a centrifugal fan \\ (rotor and stator) before and after the \\ appearance and disappearance of rotating stalls
}

\author{
B. Desmet \\ E.N.S.A.M. Lille
}

\footnotetext{
Notations

$N \quad$ vitesse de rotation (tours $/ \mathrm{min}$ )

$Q \quad$ débit volume $\left(\mathrm{m}^{3} / \mathrm{s}\right)$

$R_{2} \quad$ rayon extérieur de la roue $(\mathrm{m})$

$p \quad$ pression $(\mathrm{Pa})$

$p_{t} \quad$ pression totale $(\mathrm{Pa})$

$P_{a} \quad$ puissance sur l'arbre $(W)$

$X_{1}$ amplitude relative du premier dépassement de la réponse du circuit de mesure des pressions à un échelon

$T$ période des oscillations de la réponse du circuit de mesure des pressions à un échelon (s)

$U \quad$ vitesse d'entraînement $(\mathrm{m} / \mathrm{s})$

$C \quad$ vitesse absolue $\left(C_{u}\right.$ : composante tangentielle; $C_{d}$ composante radiale) $(\mathrm{m} / \mathrm{s})$

$W \quad$ vitesse relative $\left(W_{u}\right.$ : composante tangentielle; $W_{d}$ composante radiale $)(\mathrm{m} / \mathrm{s})$

r.m.s. valeur r.m.s.

$V_{1}, V_{2}$ tensions de sortie des anémomètres $(V)$

$t_{0} \quad$ période de passage du décollement tournant (s)

$t_{1}$ décalage temporel des tensions de sortie des anémomètres $(\mathrm{s})$

$t_{2}$ temps de passage du décollement tournant (s)

$C_{12}$ fonction d'intercorrélation des tensions $V_{1}, V_{2}$

$$
\left(C_{12}(\tau)=\lim _{T \rightarrow \infty} \frac{1}{T} \int_{0}^{T} V_{1}(t) \cdot V_{2}(t+\tau) \cdot d t\right)
$$

$i \quad$ nombre de zones décollées

$f \quad$ fréquence de rotation de la roue $(\mathrm{Hz})$

$f_{r}$ fréquence de rotation du décollement tour$\operatorname{nant}(\mathrm{Hz})$
}

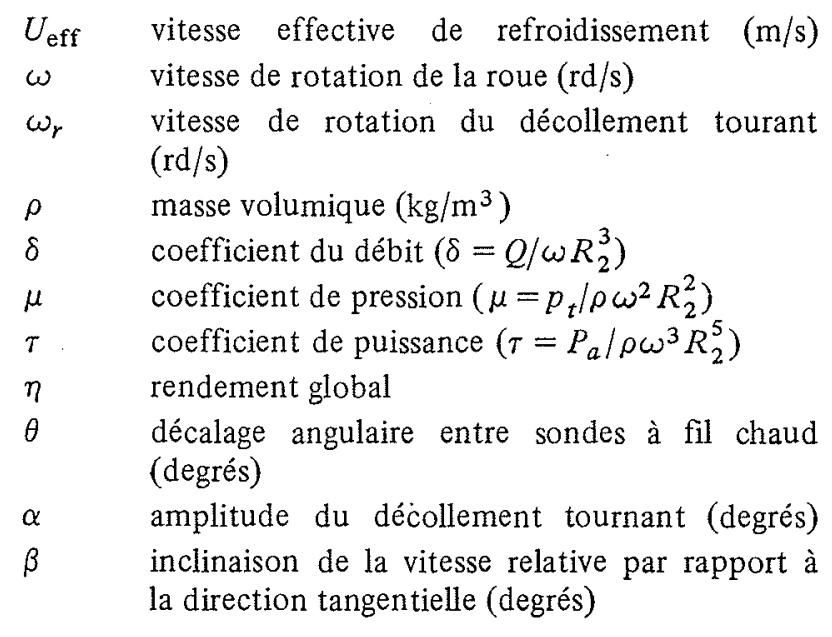

\section{Introduction}

L'exploitation des turbomachines à débit partiel s'accompagne de phénomènes tels que recirculations, décollements tournants, pompage, dont les conséquences sont néfastes pour le bon fonctionnement des installations (instabilités de fonctionnement, vibrations, bruit...). Dans le but d'améliorer la connaissance de ces phénomènes, des mesures de l'écoulement instationnaire dans un ventillateur centrifuge, en régime stable ou en présence de décollement tournant, ont été entreprises au laboratoire de mécanique de l'ENSAM Lille. Des mesures de pressions sur les aubages de la roue ont été réalisées sur toute la largeur d'un canal. Des anémomètres, à un ou deux fils chauds, ont permis la détermination de certaines caractéristiques des décollements tournants et des profils des vitesses à la sortie de la roue.

LA HOUILLE BLANCHE/N ${ }^{\circ}$ 2/3-1982 


\section{Installation d'essais}

Le ventilateur utilisé pour les essais est muni d'une roue centrifuge à simple ouie d'aspiration montée en caisson et à axe horizontal. Les principales dimensions sont données sur la figure 1 . $\mathrm{La}$ roue est munie de

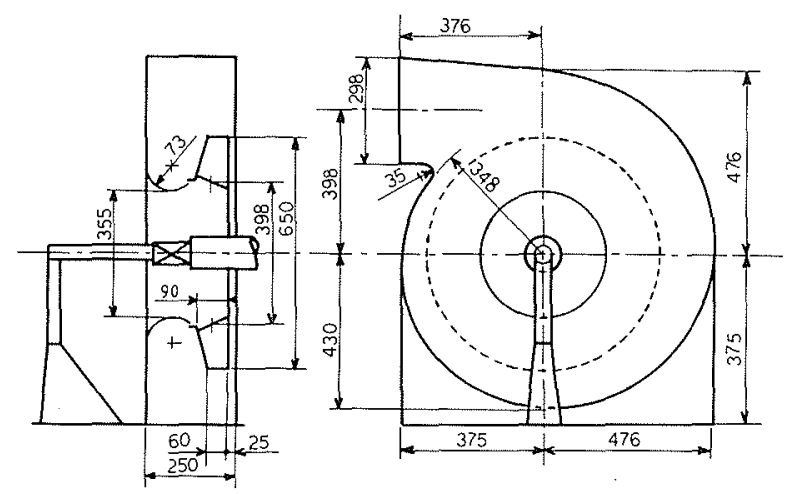

Figure 1 - Schéma du ventilateur.

16 aubages rectilignes dont l'angle de sortie, par rapport à la direction tangentielle, est $57^{\circ}$. Le rayon à l'entrée des aubages n'est pas constant, comme on peut le voir sur la figure 1 . Les aubages sont constitués par des profils NACA 16-009. Ce profil a été choisi pour sa grande épaisseur relative, ce qui est favorable pour loger des prises de pression dans l'aubage. C'est un profil laminaire de petit rayon au bord d'attaque et d'angle important au bord de fuite [1]. Les caractéristiques du ventilateur ont été mesurées par la méthode du caisson réduit suivant la norme NF X 10-200 [2]. Le ventilateur est entrainé directement par un moteur balance à courant continu dont on peut faire varier la vitesse de façon continue jusqu'à 3000 tours $/ \mathrm{mn}$. Les variations du coefficient de travail net $\mu$, du coefficient de puissance sur l'arbre $\tau$ et du rendement $\eta$ en fonction du coefficient de débit $\delta$ sont représentées sur la figure 2. Aux faibles débits, il existe une zone de discontinuité où on peut avoir un décollement tournant. Le décollement tournant est caractérisé par la formation, dans la roue, d'une ou de plusieurs zones où le débit est pratiquement nul. Ces zones tournent dans le même sens que le rotor mais à une vitesse plus faible. D'après Gottchalk [3], le nombre $\operatorname{Rot}=0,29$ calculé pour le

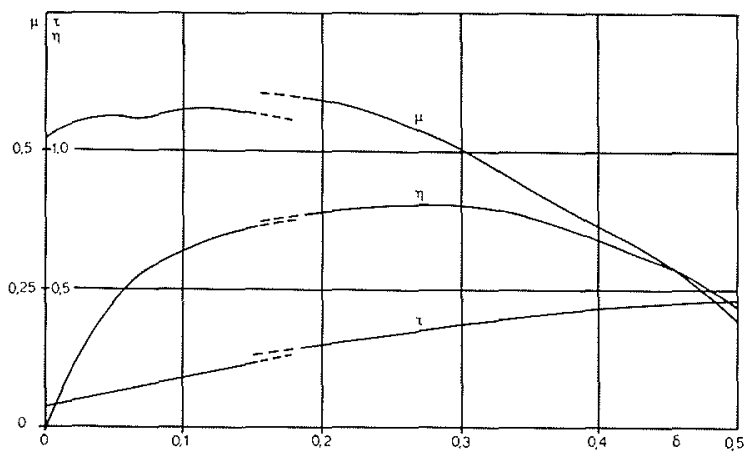

Figure 2 - Caractéristiques. débit optimal (Fig. 3) étant inférieur à la valeur critique comprise entre 0,31 et 0,37 pour un ventilateur centrifuge, il est normal de trouver un décollement tournant aux faibles débits.

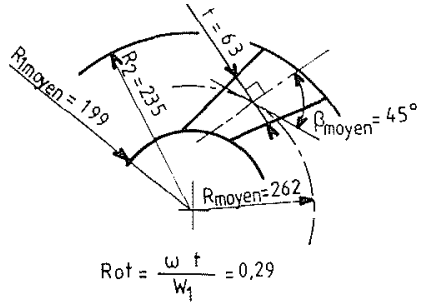

Figure 3 - Critère de décollement tournant de Gottchalk.

\section{Pressions sur aubages}

\section{Dispositif expérimental}

Les faces de deux aubages tournées vers un même canal sont munies de prises de pression dont la disposition est précisée sur la figure 4. De façon à limiter

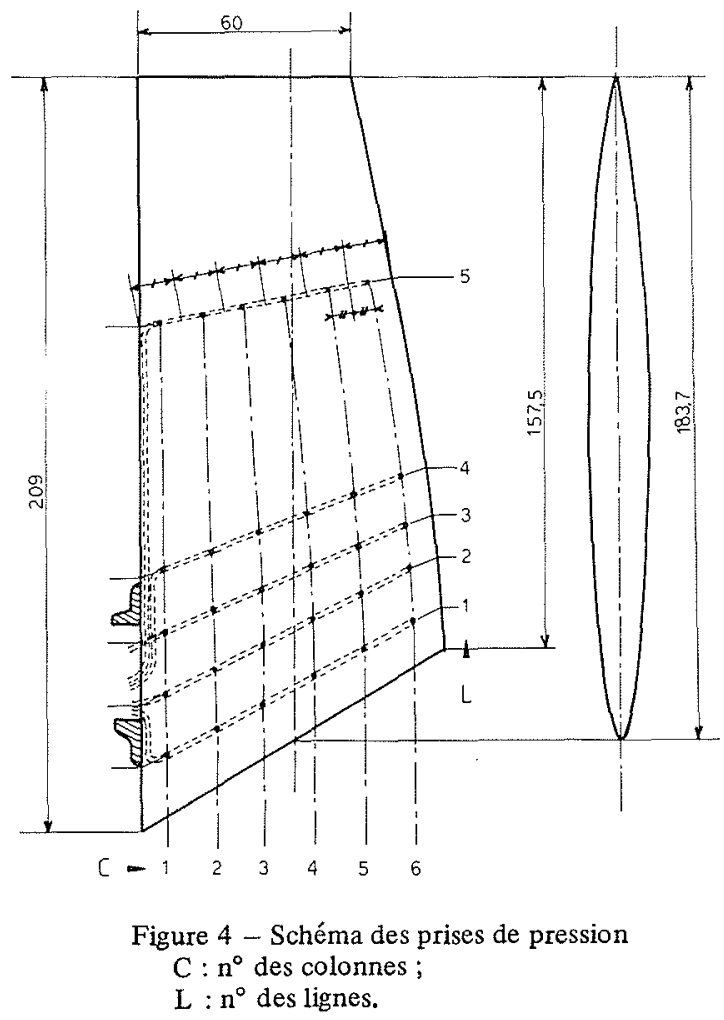

le nombre de lignes de mesure de pression, chaque ligne est connectée à six prises de pression et il est donc nécessaire d'obstruer cinq de ces prises à l'aide d'un ruban adhésif avant de faire une mesure. Les lignes de mesure de pression sont reliées à un capteur STATHAM situé hors de la roue à l'aide d'un collecteur pneumatique ONERA à 12 voies $[4,5]$. La réponse en fréquence de la chaîne de mesure (prise de pression, tube de liaison, collecteur pneumatique, capteur et pont de mesure) est obtenue à l'aide de 
la réponse temporelle du circuit soumis à un échelon de pression en assimilant le circuit à un système linéaire du second ordre [6]. La réponse est oscillante et l'amplitude relative $X_{1}$ du premier dépassement (Fig. $5 a$ ) ainsi que la période d'oscillation $\mathrm{T}$ permettent de définir les paramètres de la réponse en fréquence (Fig. 5b) [7]. La réponse en fréquence est à peu près plate jusqu'à $60 \mathrm{~Hz}$.

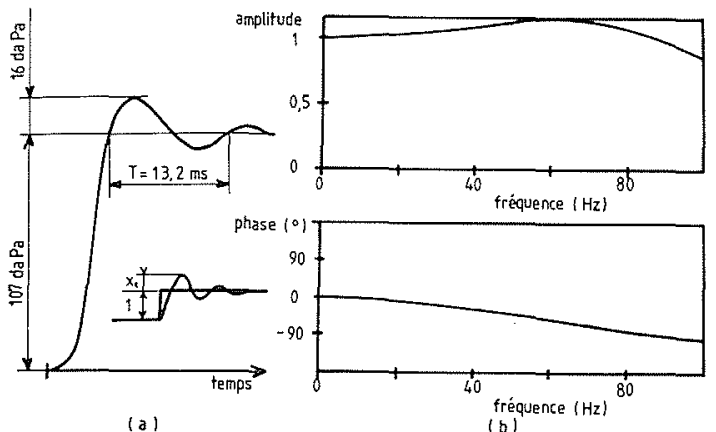

Figure 5 - Réponse en fréquence des prises de pression.

\section{Mesures réalisées}

Les pressions sur les aubages ont été enregistrées pour une vitesse de rotation du ventilateur de 1400 tours $/ \mathrm{mn}$ et un débit volume refoulé $\mathrm{Q}=0,775 \mathrm{~m}^{3} / \mathrm{s}$ en régime stable, conditions correspondant à un coefficient de débit $\delta=0,154$. A partir de ce point de fonctionnement, il est possible de faire apparaître un décollement tournant par une obstruction partielle du diaphragme de diamètre $180 \mathrm{~mm}$ placé à la sortie du caisson dans lequel débite le ventilateur. Le décollement tournant étant apparu, celui-ci persiste quand on cesse de perturber la sortie du caisson. Nous avons $\mathrm{pu}$ passer du régime de fonctionnement instable avec décollement tournant au régime stable par une obstruction partielle de l'ouie d'aspiration du ventilateur.

Les fluctuations de pression ont été enregistrées à l'entrée de l'aubage sur la face en dépression sans décollement tournant, à l'entrée et à la sortie de l'aubage, sur les deux faces, avec décollement tournant. Les enregistrements (Fig. 6), montrent, en présence du décollement tournant, que les fluctuations de pression :

- ont une amplitude plus grande qu'en régime stable à l'entrée de l'aubage sur la face en dépression;

- ont une amplitude plus grande sur la face en dépression que sur la face en pression à l'entrée de l'aubage ;

- ont des amplitudes voisines sur les deux faces vers la sortie de la roue et plus faibles qu'à l'entrée.

Des fluctuations rapides $(325 \mathrm{~Hz})$ apparaissent sur l'enregistrement de la pression sur la face en dépression à l'entrée de la roue mais la réponse limitée en fréquence du dispositif de mesure de pression ne permet pas d'interpréter ces oscillations. En l'absence de décollement tournant, l'analyse spectrale (Fig. 7) de la pression à l'entrée de la roue sur la face en dépression fait apparaître des raies à $23,6 \mathrm{~Hz}$ et $47,2 \mathrm{~Hz}$ correspondant à la vitesse de rotation de la roue. Dans

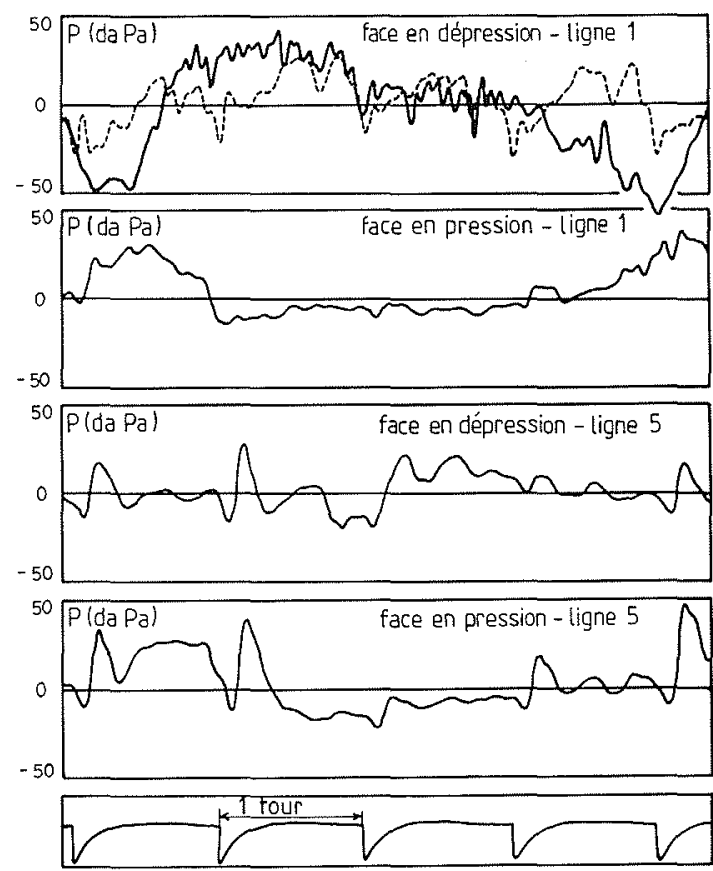

Figure 6 - Pressions sur aubage (colonne 3 ) $-N=1400$ tours/ $\min$.

$$
\begin{aligned}
& -\ldots \text { régime stable, } \\
& - \text { avec décollement tournant. }
\end{aligned}
$$

ce cas, les fluctuations de la pression sont dues aux dissymétries de l'écoulement à l'entrée et à la sortie de la roue. En présence de décollement tournant des raies supplémentaires apparaissent à $6,4 \mathrm{~Hz}, 12,8 \mathrm{~Hz}$.. Dans l'hypothèse d'une seule zone décollée, la fréquence fondamentale $6,4 \mathrm{~Hz}$ permet d'estimer la vitesse de rotation du décollement tournant par rapport à la roue à 380 tours $/ \mathrm{mn}$.
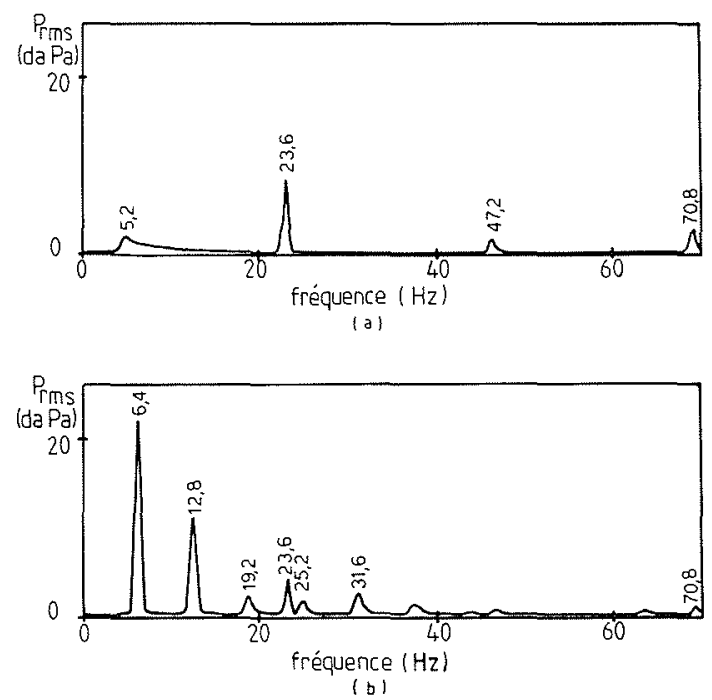

Figure 7. - Analyse spectrale des pressions sur aubage :

(a) régime stable,

(b) avec décollement tournant. 
Mesures des vitesses à l'entrée et à la sortie de la roue par anémométrie à fil chaud

\section{Sonde à un fil placée à l'entrée de la roue}

Une sonde d'anémomètre à fil chaud a été placée devant la roue au centre du canal et à $10 \mathrm{~mm}$ environ du bord d'attaque des aubages. Les vitesses ont été mesurées pour des conditions de fonctionnement identiques à celles définies au paragraphe précédent. Pour le régime stable, l'enregistrement des vitesses (Fig. 8) présente des fluctuations dues au passage des aubages. Le passage du décollement tournant produit des fluctuations de la vitesse dont l'amplitude est comparable à la vitesse moyenne à l'entrée de la roue $(6,9 \mathrm{~m} / \mathrm{s}$ en régime stable). Sur l'analyse spectrale des vitesses en régime stable (Fig.9), les raies à 23,6 Hz, 47,2 Hz. . . sont
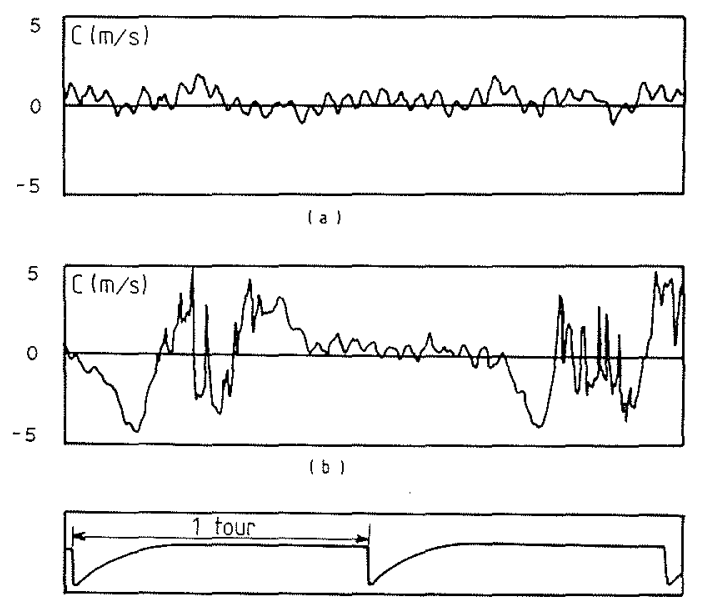

Figure 8 - Fluctuations de vitesse à l'entrée de la roue : (a) régime stable ;

(b) avec décollement tournant.
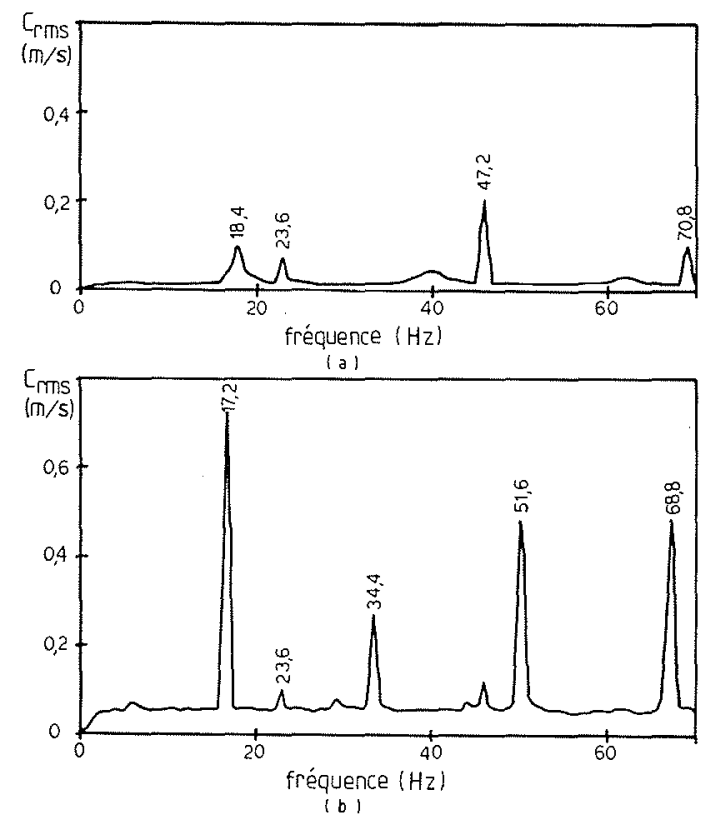

Figure 9 - Analyse spectrale des vitesses :

(a) régime stable ;

(b) avec décollement toumant. dues aux dissymétries de la roue. En présence du décollement tournant, des raies d'amplitudes plus importantes apparaissent à $17,3 \mathrm{~Hz}, 34,4 \mathrm{~Hz}, 51,6 \mathrm{~Hz}$. . S'il n'existe qu'une seule zone décollée dans la roue, la fréquence fondamentale $17,2 \mathrm{~Hz}$ permet d'évaluer la vitesse de rotation absolue du décollement tourant à 1030 tours/mn, ce qui est en bon accord avec la vitesse de rotation relative du décollement de 380 tours/ mn trouvée à partir de l'analyse spectrale des pressions sur l'aubage. Une analyse spectrale autour de la fréquence de passage des aubages $377,6 \mathrm{~Hz}$ (Fig. 10) comporte, en présence de décollement tournant, des bandes latérales espacées de $17,2 \mathrm{~Hz}$ correspondant à la modulation du passage d'aubages par le décollement tournant.
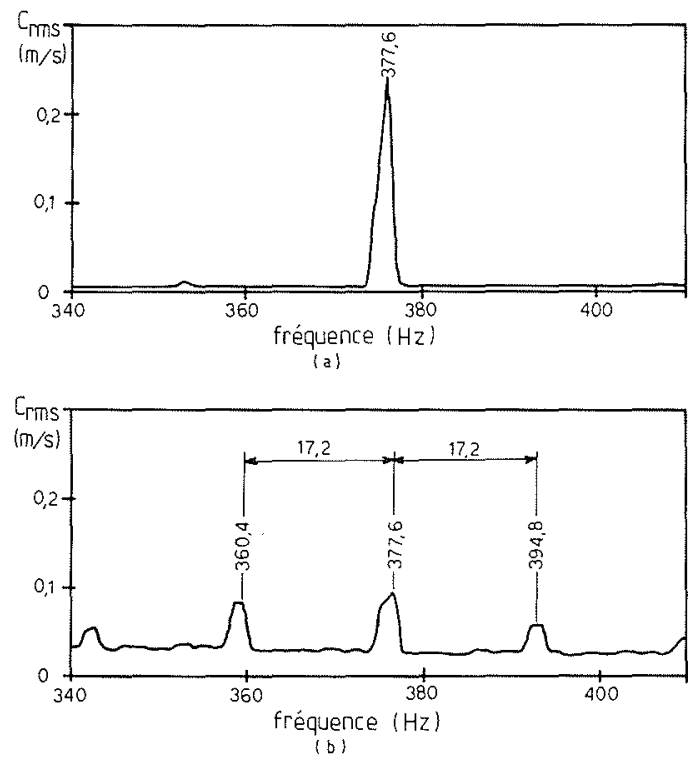

Figure 10 - Analyse spectrale des vitesses :

(a) régime stable;

(b) avec décollement tournant.

Utilisation de deux sondes à un fil à l'entrée de la roue

Deux fils chauds sont placés à l'amont de la roue $[8,9]$, assez près de l'entrée des aubages pour détecter l'apparition du décollement tournant, avec un décalage angulaire $\theta$ (Fig. $11 a$ ). Les sorties des deux anémomètres sont reliées à un analyseur de signaux Hewlett-Packard HP 5423. Une schématisation des signaux foumis par les anémomètres est donnée par la figure $11 b$. On en déduit les caractéristiques suivantes du décollement tournant :

- vitesse de rotation par rapport à un repère fixe

$$
\omega_{r}=\pi \cdot \frac{\theta}{180} \cdot \frac{1}{t_{1}}
$$

avec $\omega_{r}$ en $r d / s ; \theta$ en degrés et $t_{1}$ en $s$.

- le nombre de zones décollées

$$
i=\frac{360}{\theta} \cdot \frac{t_{1}}{t_{0}}
$$




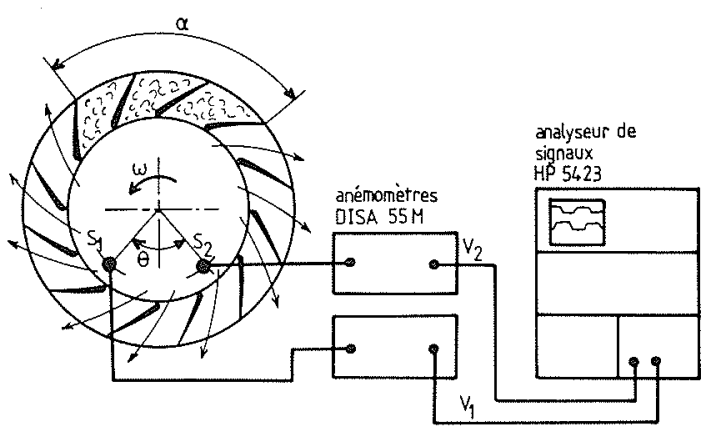

(a)

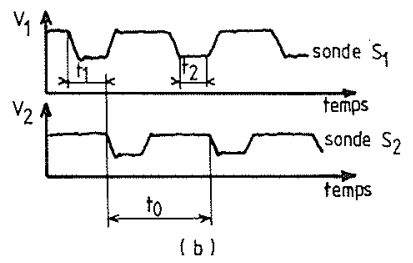

Figure 11 - Analyse du décollement tournant à l'aide de 2 fils chauds :

(a) dispositif expérimental ;

(b) schématisation des signaux obtenus à l'entrée d'un compresseur axial d'après [7].

- l'amplitude d'une zone décollée

$$
\alpha=\theta \cdot \frac{t_{2}}{t_{1}}
$$

avec $\alpha$ en degrés

En pratique, les signaux de sortie des anémomètres comportent un bruit important (Fig. 12) et la détermination directe des temps $t_{0}$ et $t_{1}$ est très imprécise. A l'aide de l'analyseur de Fourier rapide, en utilisant la fonction d'intercorrélation pour les tensions de sortie $V_{1}$ et $V_{2}$ des deux anémomètres, il est possible d'accéder aux temps $t_{0}$ et $t_{1}$ avec une meilleure précision. La figure 13 montre l'évolution du rapport entre la fréquence de rotation du décollement tournant et celle de la roue en fonction du coefficient de débit $\delta$ et précise le nombre de zones décollées. Nous avons également vérifié que ces caractéristiques n'évoluent pas quand la vitcssc de rotation de la rouc varic de 1000 à 2500 tours/mn.

\section{Sonde à deux fils croisés à la sortie de la roue}

La sonde utilisée, à deux fils croisés à $90^{\circ}$ (Fig. 14), a été étalonnée en vitesse et en direction dans une soufflerie. Cette sonde a été placée derrière la roue au rayon $337 \mathrm{~mm}$, ce qui correspond à une distance du bord de fuite des aubages égale à $12 \mathrm{~mm}$. La chaîne de mesure des vitesses est représentée sur la figure 15 . L'analyseur de signaux HP 5423 est utilisé comme mémoire numérique de capacité 512 points sur chacune des deux voies. Pour la fréquence d'échantillonnage maximale la durée d'une acquisition est de $5 \mathrm{~ms}$. Le stroboscope avec unité de déclenchement fournit une impulsion par tour et synchronise le début de
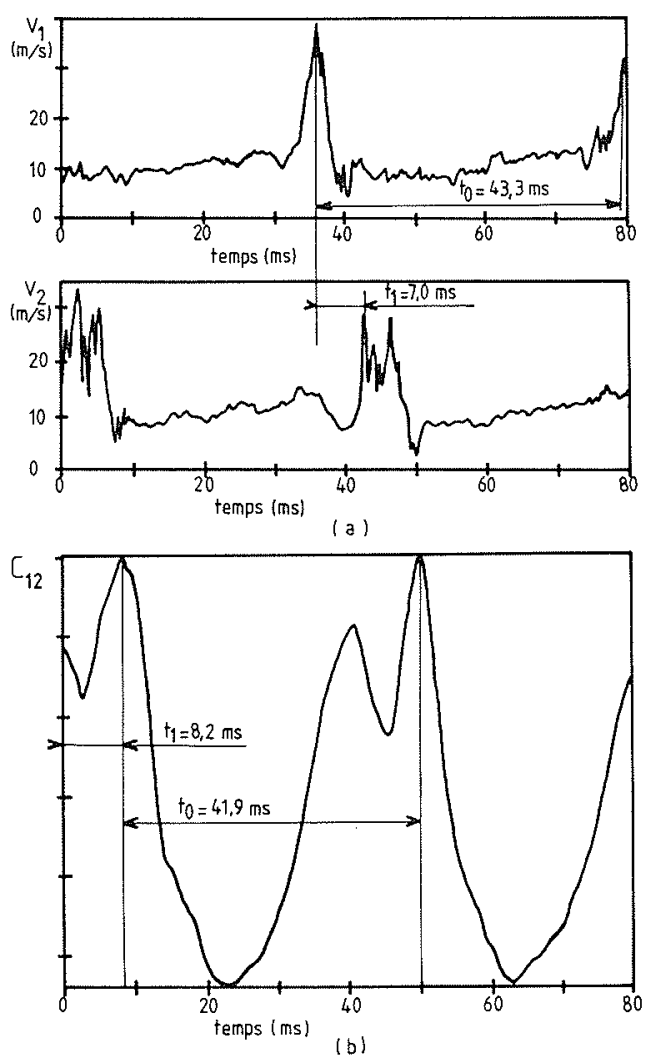

Figure 12 - Analyse du décollement tournant à l'aide de 2 fils chauds $-\mathrm{N}=1985$ tours $/ \mathrm{min}$.

(a) signaux de sortie des anémiomètres;

(b) intercorrélation des signaux de sortie ( $V_{1}$ retardé).

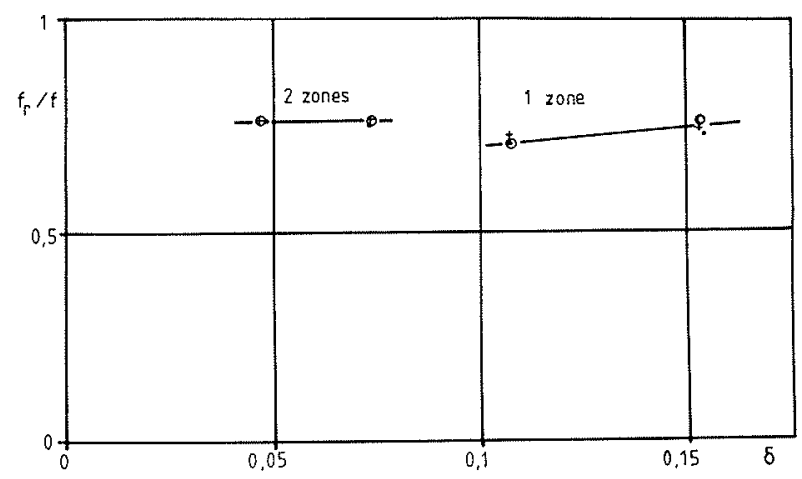

Figure 13 -- Caractéristiques du décollement toumant :

$f_{Y}$ : fréquence de rotation du décollement;

$f$ : fréquence de rotation de la roue ;

- 1000 tours $/ \mathrm{min}+1500$ tours $/ \mathrm{min} \quad 2000$ tours $/ \mathrm{min}$.

l'acquisition des signaux foumis par les anémomètres sur le passage d'un même aubage, permettant ainsi d'ob. tenir une loi moyenne de la distribution des vitesses d'aube à aube dans un canal et pour une position angulaire fixée de la roue. Les composantes moyennes et fluctuantes des sorties des anémomètres sont enregistrées séparément pour améliorer la résolution d'acquisition des composantes fluctuantes. Le calculateur HP 9835 pilote l'ensemble de la procédure d'acquisition des signaux des anémomètres. Il permet, à partir des lois d'étalonnage des fils chauds, un calcul 


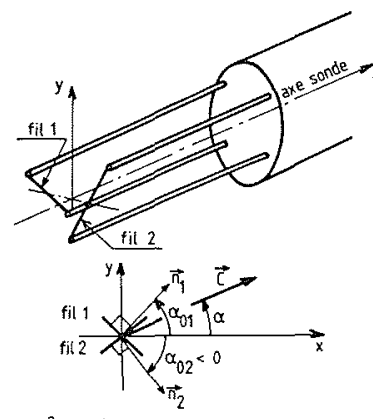

$u_{\text {eff }}^{2}=\left[^{2} \cdot\left(\cos ^{2}\left(\alpha_{0}-\alpha\right)+k^{2} \cdot \sin ^{2}\left(\alpha_{0}-\alpha\right)\right]\right.$

(a)

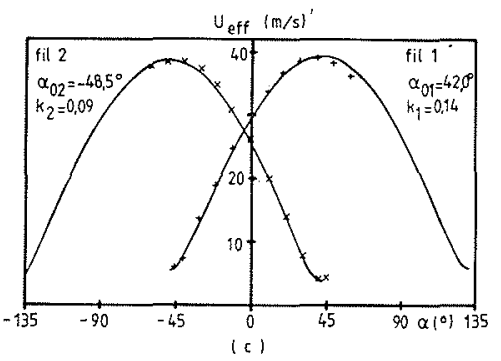

Figure 14 - Sonde à 2 fils chauds :

(a) schéma de la sonde;

(b) étalonnage en vitesse ;

(c) étalonnage directionnel.

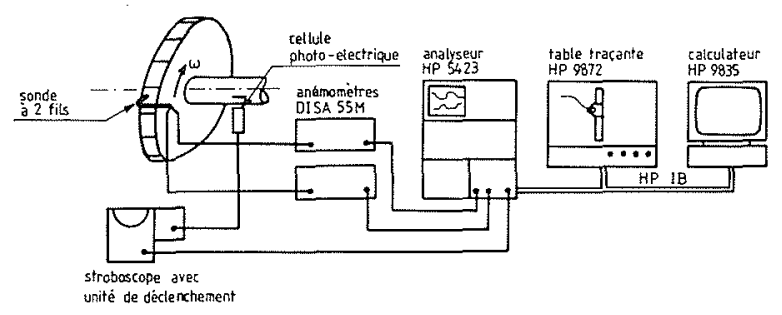

Figure 15 - Mesure des vitesses à l'aide d'une sonde à 2 fils Dispositif expérimental.

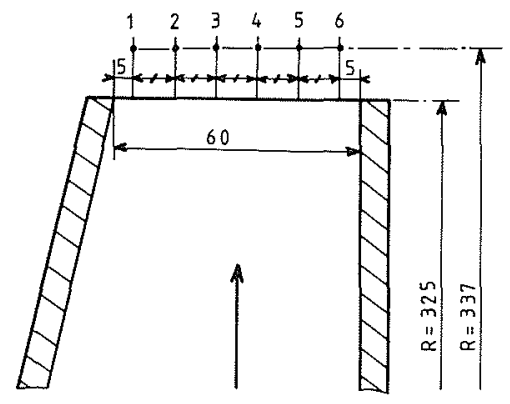

Figure 16 - Situation des points de mesure des vitesses.

immédiat des vitesses qui sont ensuite représentées sur l'écran de l'analyseur HP 5423 et qui peuvent être enregistrées sur l'unité de bande magnétique du calculateur en vue d'un traitement ultérieur. La distribution des vitesses, aube à aube, a été relevée en six points sur la largeur des canaux (Fig. 16). Les figures 17,18 , et 19 montrent des exemples de distributions de vitesses obtenues pour une vitesse de rotation de la roue de 2000 tours $/ \mathrm{mn}$ et au point de meilleur rendement $\left(Q=2,0 \mathrm{~m}^{3} / \mathrm{s}\right)$.
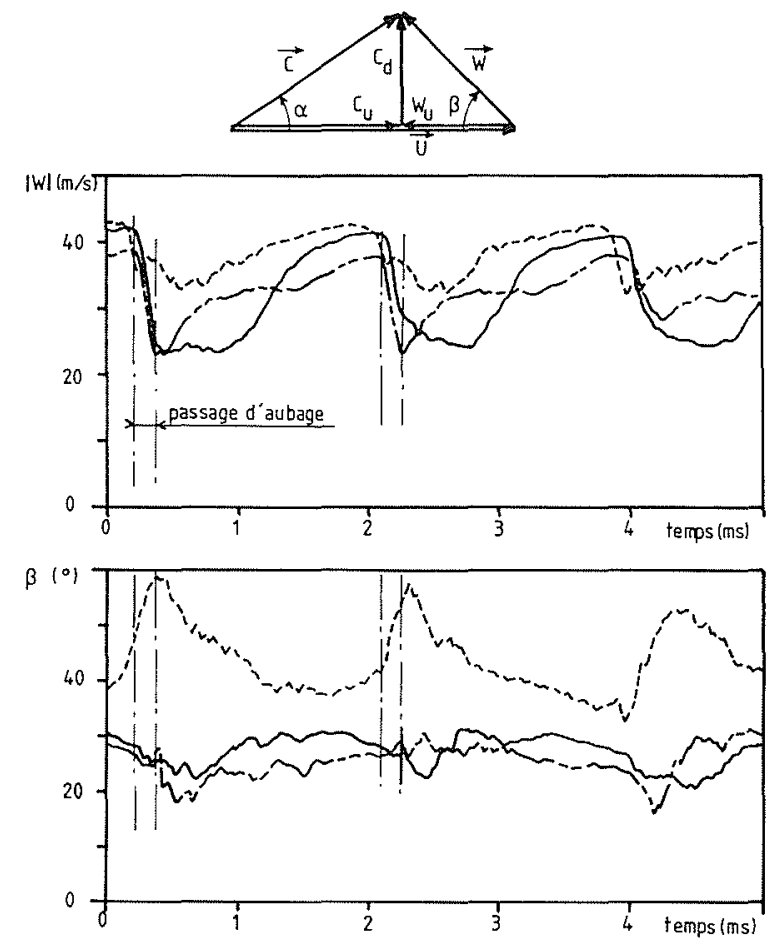

Figure 17 - Vitesses derrière la roue $-\mathrm{N}=2000$ tours/min $Q=2 \mathrm{~m}^{3} / \mathrm{s}$

- - point $1 \longrightarrow$ point $3--$ point 6 .

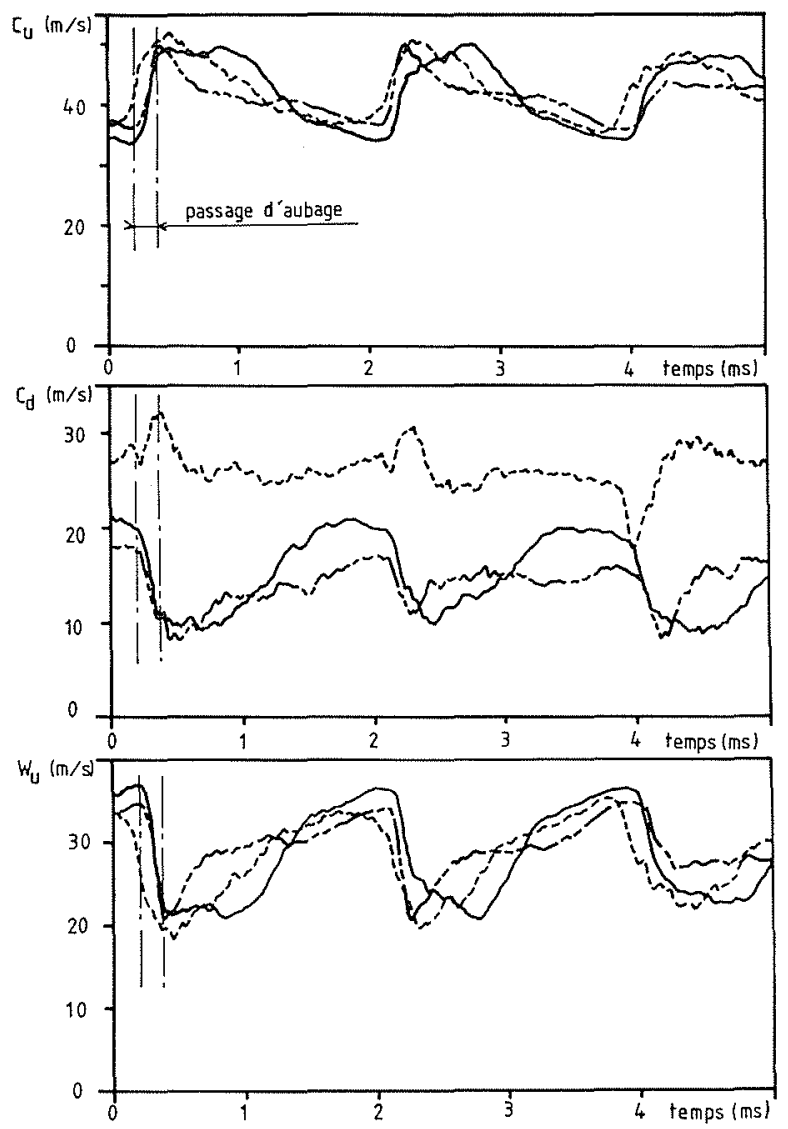

Figure 18 - Vitesses derrière la roue $-\mathrm{N}=2000$ tours $/ \mathrm{min}$ $Q=2 \mathrm{~m}^{3} / \mathrm{s}$ :

- - point $1 \longrightarrow$ point $3-\ldots$ point 6 . 


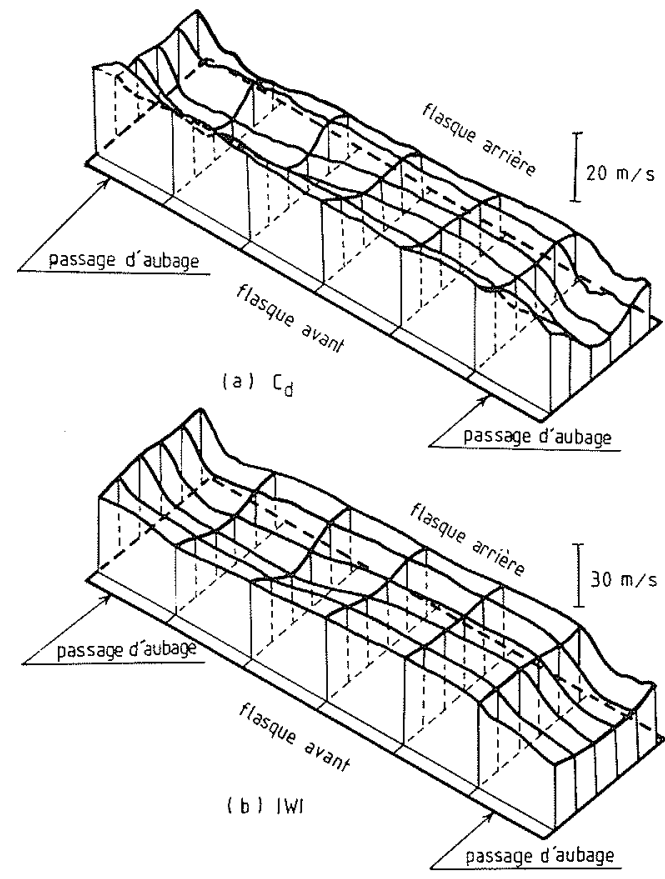

Figure 19 - Vitesses derrière la roue $-\mathrm{N}=2000$ tours/min $Q=2 \mathrm{~m}^{3} / \mathrm{s}$;

(a) vitesse radiale ;

(b) vitesse relative.

Pour ce point de fonctionnement :

- les fluctuations de la vitesse débitante $C_{d}$ sont plus atténuées près des flasques et surtout vers le flasque avant ;

- la vitesse débitante est plus élevée vers le flasque avant ;

- la structure d'écoulement avec jet et zone décollée vers la face en dépression des aubages est plus marquée dans la zone centrale des canaux.

\section{Conclusions}

Les pressions sur aubages, les mesures à l'aide de un ou deux fils chauds placés à l'entrée de la roue permettent de connaître les caractéristiques suivantes : vitesse de rotation du décollement tournant, nombre et étendue des zones décollées. Par utilisation d'un analyseur rapide de Fourier (fonction d'intercorrélation) ces résultats peuvent ètre obtenus avec une bonne précision.

La connaissance de la distribution des vitesses radiales et tangentielles sur la largeur du canal à la sortie de la roue permet de définir le facteur de glissement [10].

Des mesures complémentaires à l'aide de sondes à 2 ou 3 fils chauds, placées à l'entrée et à la sortie de la roue, en synchronisant l'acquisition des signaux sur le passage d'un aubage et sur la présence du décollement tournant, doivent permettre une meilleure connaissance de la structure du décollement toumant et des conditions d'apparition de celui-ci.

\section{Bibliographie}

[1] ABBOT I.H. VON DOHENHOFF R.E. - Theory of wing sections; Dover Publications, New York.

[2] Norme française NF X 10-200; Règles d'essais aérauliques en plate-forme des ventilateurs à enveloppe; juin 1971

[3] GOTTCHALK M. - Untersuchung der Kennlinien-stetigkeit von Radial Ventilatoren; Mitteilungen des Instituts für Strömungslehre und Strömungsmachinen; Universität (TH) Karisruhe ; Heft 17 ; September 1974.

[4] LARGUIER R., SIEVERS A. - Méthodes de mesures instationnaires dans les turbomachines; Aéronautique et Astronautique ; $\mathrm{n}^{\circ} 46 ; 1974-3 ;$ p. 9-18.

[5] LAKSHMINARAYANA B. - Techniques for aerodynamic and turbulence measurements in turbomachinery rotors; Measurements methods in rotating components of turbomachinery. Joint fluids engineering gas turbine conference and product show ; New-Orléans; March 10-13; 1980 ; p. 5-30.

16] DAMION J.P. - Application de l'étalonnage dynamique à la mesure des pressions variables; Bulletin du BNM; $\mathrm{n}^{\circ} 38$; octobre 1979 ; p. 11-19.

[7] GILLE, DECAULINE, PELEGRIN. - Théorie et calcul des asservissements; Dunod 1963.

[8] DUDZISZ J. - Experimental investigations of the influence of compressor cascades interaction on the rotating stall phenomenon; Proceedings of the 3rd conference onf fluid machinery; Akademiai Kiado; Budapest 1969 ; p. 115-121.

[9] SUZUKI, UGAI, MARADA. - Noise characteristics in partial discharge of centrifugal fans; Bull JSME; vol. 21 ; $\mathrm{n}^{\circ} 154$; April 1978 ; p. 689-696.

[10] MURAKAMI M., KIKUYAMA K, ASAKURA E. - Velocity and pressure distributions in the impeller passages of centrifugal pumps; $A S M E$ trans., Journal of fluids engineering; vol. $102 ; \mathrm{n}^{\circ} 4$; December $1980 ;$ p. $420-426$

\section{Discussion}

Président : J. PAULON

Le Président. - Je remercie M. DESMET pour cet exposé très clair.

M. SCHIA VELLO. - Quelle est la forme des aubes?

M. DESMET. - Ces aubes sont planes, mais profilées. C'est un profil NACA 16.009 choisi pour sa relativement grande épaisseur, ce qui est indispensable pour loger les prises de pression.

Il y a 16 aubes, L'angle de sortie est de $57^{\circ}$. Pour l'angle d'entrée, je n'ai plus le $\beta$ en tete. Bien entendu, il varie puisque le rayon d'entrée n'est pas toujours le même.

M. SCHIAVELLO. - Quel est le rapport entre la corde et le pas moyen?

M. DESMET. - Javoue que je ne le connais pas. Il faut reprendre la figure 3 du rapport et le calculer. Vous avez là les dimensions de l'aubage au flasque avant et au flasque arrière. 
M. SCHIAVELLO. - Il serait intéressant de voir la distribution de l'angle de l'écoulement relatif entre la ceinture et le moyeu. Est-ce qu'on peut avoir la distribution géométrique de l'angle $\beta$.

Le point 3 correspond-il à la ligne moyenne ?

M. DESMET. - C'est ce qui correspond à la zone centrale de l'aubage. La courbe supérieure correspond au flasque avant; la courbe en trait plein à la zone moyenne; et la courbe en-dessous au flasque arrière.

M. SCHIAVELLO. - Si on regarde le point 1 (flasque avant) on voit que l'angle $\beta$ est plus grand. C'est un effet d'écoulement secondaire à la ceinture des aubes; c'est l'effet d" "over-turning".

Ce qui ne correspond pas à la théorie, c'est que l'angle $\beta$ sur le flasque du haut doit être aussi, en principe, plus élevé que l'angle sur la ligne moyenne. C'est déjà indicatif du fait que l'aube à la ceinture est plus chargée par l'effet des écoulement secondaires.

Il serait intéressant de voir quelles sont les conditions de "solidity" et de faire une tentative de calcul de l'effet secondaire et des déviations additionnelles par écoulement secondaire.

M. KECK. - Quelqu'un a-t-il l'expérience de mesures analogues dans l'eau, et non plus dans l'air? Pensez-vous comme moi que ces mesures sont très importantes si l'on veut essayer de calculer ces écoulements compliqués.

M. DESMET. - En ce qui concerne les mesures dans l'eau. j'ai vu récemment un article paru dans "Journal of fluids Engineering". Je dois pouvoir vous l'adresser si cela vous intéresse;

Disons que le but de ces mesures est surtout d'aller voir ce qui se passe quand on se trouve en présence de fonctionnements instables, donc d'essayer de préciser quelles sont les conditions d'apparition des décollements tournants en tentant de faire varier progressivement le régime pour s'approcher des zones où peuvent apparaître des décollements tournants, et regarder comment évolue l'écoulement quand on s'approche de ces zones. C'est dans un but de recherche de l'apparition des conditions de décollement tournant plutôt que pour effectuer des calculs que l'on a été amené à développer ce genre de technique de mesures.

M. ANDRE. - Je voudrais répondre à M. KECK, en tant qu'ancien constructeur de gros ventilateurs pour centrales thermiques.
Nous avons été intéressés par les recherches sur le décollement tournant dans les hélicoides et dans les centrifuges et également par les travaux que le Professeur JAUMOTrE a fait sur ce sujet à l'Université de Bruxelles.

En ce qui concerne les machines axiales, il y a une rotation qui se fait en sens inverse à la moitié de la vitesse du ventilateur. On observe des phénomènes de remplissage et de désemplissage entre chaque aube, entraînant des phénomènes de pulsation.

J'estime que ces recherches sont très intéressantes pour les constructeurs.

M. FRADIN. - La vitesse de rotation des zones décollées parait supérieure à ce que l'on trouve habituellement dans la littérature. La continuité de cette étude, en vue d'analyser le phénomène de décollement tournant et les critères conduisant à son apparition, paraît souhaitable sur ce montage compte tenu des facilités d'accès et de l'instrumentation déjà mise en œuvie.

Il est cependant souhaitable d'étudier l'écoulement du fluide à un débit très légèrement supérieur au débit d'apparition du décollement tournant. L'instrumentation déjà mise en cuvre peut être avantageusement complétée par l'adjonction de capteurs pelliculaires développés à l'ONERA et de sondes de pression d'arrêt liées au rotor.

Compte tenu des vitesses de rotation modérées je ne crois pas qu'il $\mathrm{y}$ ait des problèmes mécaniques. Ainsi vous verriez les zones de fluctuation de débit liées aux zones de fluctuations de pression.

Lorsqu'on met un capteur de pression sur la paroi avant et qu'on mesure ces fluctuations de pression (qui sont très importantes, puisqu'elles peuvent aller jusqu'à $20 \%$ de ta pression statique à la sortie du rotor), on voit apparaître par moment des fluctuations de pression liées à la force de Coriolis. On peut en déduire que dans ces régions il passe du fluide alors que dans la demi-période suivante, il ne passe plus rien.

Je crois que c'est très intéressant à voir, avec du matériel plus performant précisément.

Le président. - Je remercie M. FRADIN de parler des travaux faits à l'ONERA en ce domaine, et de signaler l'intérêt des capteurs pelliculaires. Evidemment cela sort un peu du sujet; mais je signale aux personnes qui pourraient être intéressées qu'il existe des capteurs pratiquement sans épaisseur qui peuvent se coller sur les aubes sans qu'il y ait à les modifier et qui permettent de faire des mesures aussi bien dans l'air que dans l'eau.

\section{Abstract \\ Pressure and speed measurements in a centrifugal fan (rotor and stator) before and after the appearance and disappearance of rotating stalls}

A centrifugal fan research facility, designed for pressure measurements on impeller blades is described in this paper. At low flow rates, a zone of discontinuity can be observed in the variations of head coefficient $\mu$, power coefficient $\tau$ and efficiency $\eta$ with the flow coefficient $\delta$. A rotating stall may occur in this area.

The frequency response of the blade pressure measuring device has been determined experimentally, by using the time response to a pressure step. The blade pressure fluctuations increase when the rotating stall appears, especially on the suction side at the inlet of the blade. The relative speed of the rotating stall has been determined by carrying out a frequency analysis of pressure fluctuations.

The absolute speed of the rotating stall has also been determined by the frequency analysis of the signal of a hotwire anemometer at impeller inlet. A bette knowledge of the rotating stall (speed, number and amplitude of the stall zones) can be reached by using two hot-wire anemometers on two radii near the inlet of the impeller. The determination of the periodicity of the anomemeters' output signals and their time shifting can be determined by using the intercorrelation function. The frequency and the number of stall zones with the variations of the flow coefficient $\delta$ have been studied. The fact that those characteristics do not change when the speed of the fan changes from $1000 \mathrm{rpm}$ to $2500 \mathrm{rpm}$ has been verified.

The blade to blade velocity distribution on the whole width of the channel has been obtained by using a probe with two hot wires at impeller outlets. The flow structure with jet and wake can be observed at the centre of the channel and the flow is more uniform near hub and shroud. 